\title{
SUBMICRON X-RAY DIFFRACTION
}

\author{
A.A.MacDowell ${ }^{1}$, R.S.Celestre ${ }^{1}$, N.Tamura ${ }^{1}$, R.Spolenak ${ }^{2}$, B.Valek ${ }^{3}$, W.L.Brown ${ }^{2}$, \\ J.C.Bravman $^{3}$, H.A.Padmore ${ }^{1}$, B.W.Batterman ${ }^{1}$ \& J.R.Patel ${ }^{1,4}$ \\ ${ }^{1}$ ALS/LBL, 1 Cyclotron Road, Berkeley, CA 94720, USA \\ ${ }^{2}$ Bell Laboratories, Lucent Technologies, Murray Hill, NJ 07974, USA \\ ${ }^{3}$ Dept. of Mat. Sci. \& Eng., Stanford University, Stanford, CA 94305, USA \\ ${ }^{4}$ SSRL/SLAC, Stanford University, Stanford, CA 94309, USA
}

\begin{abstract}
At the Advanced Light Source in Berkeley we have instrumented a beam line that is devoted exclusively to $\mathrm{x}$-ray micro diffraction problems. By micro diffraction we mean those classes of problems in Physics and Materials Science that require x-ray beam sizes in the sub-micron range. The instrument is for instance, capable of probing a sub-micron size volume inside micron sized aluminum metal grains buried under a silicon dioxide insulating layer. The resulting Laue pattern is collected on a large area CCD detector and automatically indexed to yield the grain orientation and deviatoric (distortional) strain tensor of this sub-micron volume. A four-crystal monochromator is then inserted into the beam, which allows monochromatic light to illuminate the same part of the sample. Measurement of the diffracted photon energy allows for the determination of $d$ spacings. The combination of white and monochromatic beam measurements allow for the determination of the total strain/stress tensor (6 components) inside each sub-micron sized illuminated volume of the sample.
\end{abstract}

\section{Keywords}

X-ray micro-diffraction, electromigration, x-ray focusing,

Contact Author Alastair MacDowell, Lawrence Berkeley National Lab., MS 2-400, Berkeley, Ca 94720, tel. 510486 4276, fax 5104867696 e-mail:aamacdowell@lbl.gov

\section{Introduction}

X-ray diffraction is a technique that has been used for about a century for elucidating the structure of materials on the macroscopic scale $(0.1-10 \mathrm{~mm})$. With the increasing need from industry to develop materials of high mechanical performance, a good understanding of their properties at the mesoscopic scale $(0.1-10 \mu \mathrm{m})$ has become critical since many of these properties are dependant on the behavior of structural entities at this scale (grain boundaries, inclusions, intrinsic intra- and inter-granular stress distribution). There is a significant amount of complexity when dealing with the mutual interactions of a large number of mesoscale grains especially when dislocations are involved. The situation is influenced by such parameters as mechanical stress, temperature, and contact with other materials. The limited amount of experimental data is due to the lack of a suitable technique. This has long prevented modeling material behavior at this length scale, which in turn has prevented progress in developing a systematic link of material properties from the macroscopic to the microscopic. With the recent availability of bright third generation synchrotron sources and progress in $\mathrm{x}$ ray focusing optics, it is now practical to develop x-ray diffraction and apply it on the micron scale length to measure the mesoscopic properties of materials. 
The ability to accurately characterize the orientation and strain/stress state in individual grains of a sample is a basic requirement to understand how materials behave at the mesoscopic scale. Using x-rays as a microprobe also allows the characterization of buried grains under overlying cap layers and multilayered films without the need of any sample preparation. This lack of sample preparation is especially important since the sample stress state can be greatly affected by any preparation process. This can be a major drawback of the electron micro probe techniques. The accurate measurement of strain gradients at the micron and sub-micron level will be useful for solving many scientific and technological problems. These range from the strain state under nano-indenters to gradients at crack tips of turbine blades and artificial heart valves. Undoubtedly many other applications will unfold in the future as X-ray micro diffraction is developed at other storage rings around the world.

In this work we describe a recently built beamline that can measure the grain orientation and triaxial strain in an arbitrarily orientated micro crystal in a buried thin film. As a demonstration of this technique, we addressed a practical and current problem in the semiconductor industry. The sub-micrometer wires which interconnect the active transistor elements in an integrated circuit can be deleteriously affected by a phenomenon called electromigration. As device structures and their interconnecting wires are made smaller and smaller, electrical current densities in the interconnects (which are increasing from $10^{5}$ $\mathrm{Amp} / \mathrm{cm}^{2}$ toward $10^{6} \mathrm{Amp} / \mathrm{cm}^{2}$ ) actually transport the atoms of the wires themselves, inducing voids and hillocks to form and consequent failure of the device. Experiments have shown that the microstructure of these wires significantly affect this process. For example, a favorable stress build up in the wire can impede or stop electromigration [1,2]. In spite of much effort in this field $[3,4,5]$, the relation between microstructure (grain orientation, grain boundaries, residual stress state, impurities, interfacing with other compounds, etc) and electromigrationinduced failure is still not understood to a great depth. Our intent is to provide new information on the electromigration phenomenon by investigating the mesoscopic physics by way of this new x-ray microdiffraction instrument.

\section{Instrumental Considerations}

The customary way of performing x-ray diffraction is to fix the photon energy and map the Bragg reflection peaks by rotating the sample while detecting the diffracted x-rays with a detector. Such a scheme is inappropriate for micron-sized samples since current high quality diffractometers have a sphere of confusion of $\sim 10 \mu \mathrm{m}$ and any rotation would move the sample out of the micro beam. In our arrangement the sample remains fixed except for translation motion in the plane of the sample. The detector can be rotated into an appropriate position around the sample. The sample is illuminated with focused white radiation and the diffracted $\mathrm{x}$-rays are recorded on a CCD detector as a Laue diffraction pattern. This can be indexed from a known or trial structure to give crystal orientation. Then by scanning the photon energy and knowing the direction of the Laue beams and crystal orientation, d-spacing measurements can be made. For crystal orientation and strain measurements the x-ray optical system must fulfill the following requirements.

1. Focus both white and monochromatic light down to a sub micron sized focus.

2. Switch between white and monochromatic x-rays without changing the point on the sample being irradiated.

3. The ability to scan in energy whilst illuminating the same point on the sample. 
The schematic layout of the beamline shown in fig. 1 is able to perform these tasks and will now be described.

\section{Beamline Description and Performance}

As the $\mathrm{x}$-ray source the beamline uses a bending magnet at the Advanced Light Source (ALS) ( $1.9 \mathrm{GeV}, 1.27 \mathrm{Tesla}, 400 \mathrm{~mA}) .3 \times 0.2 \mathrm{mrads}$ of light is collected by a platinum coated silicon toroidal mirror [6] operating at a grazing angle of 5.4mrads in the 1:1 focusing condition. The mirror focuses light to the front on the hutch at the location of some XY x-ray slits that are adjustable in size. Beyond these slits is a $2.5 \mathrm{~m}$ long section within the hutch that is occupied by a time resolved x-ray diffraction station, followed by the micro diffraction end station. This end station consists of a four-crystal monochromator followed by 2 platinum coated orthogonal Kirkpatrick Baez (KB) micro focusing mirrors [7], the sample and a 90x90mm active area X-ray CCD [8]. The sample and CCD are mounted on a 6-circle Huber goniometer. The schematic of the engineering model used for the end station instrument is shown in fig. 2 .

The slits at the front of the hutch act as an adjustable sized source for the KB mirrors of the micro-diffraction station. In this way spot size can be traded for flux. The 4-crystal monochromator has the property of either passing white light or monochromatic light down the same axis. This allows for easy switch over between white and monochromatic light while illuminating the same point on the sample. The individual items in the beamline will now be discussed in detail.

The slits consist of two $10 \mathrm{~mm}$ diameter water-cooled polished tungsten rods that are separated by $2 \mathrm{~mm}$. By rotating the rods on a rotary vacuum mechanism any slit size can be chosen between 0 and $2 \mathrm{~mm}$ with a resolution of $\pm 1 \mathrm{~mm}$. Making the slits from $10 \mathrm{~mm}$ diameter tungsten ensures that the slit edges are well defined. The orthogonal set of slits is identical but displaced $5 \mathrm{~cm}$ downstream. The whole slit assembly can be rastered around to measure the focused beam profile.

The bending magnet source used at the ALS has a source size of approximately 250x $25 \mu \mathrm{m}$ FWHM $(\mathrm{HxV})$. In the 1:1 imaging condition a perfect toroidal mirror will faithfully image this source onto the tungsten slits. Tangential errors in the mirror will result in some reduction in vertical brightness. Focused spot sizes using the full aperture of the mirror have been measured at $250 \times 70 \mu \mathrm{m}$ FWHM. This is fine for the time resolved experiment that is mainly interested in flux. The micro-diffraction end station cannot accept all the light collected by the toroid due to aperture restrictions imposed by the KB mirrors. (to be described later). Aperturing the toroidal mirror down to about $30 \%$ of its acceptance results in a focused spot size of $250 \mathrm{x} 45 \mu \mathrm{m}$ FWHM. This implies tangential figure errors of $\sim 0.5 \mu \mathrm{rad}$ over the used part of the mirror $(15 \times 600 \mathrm{~mm})$. Brightness has been reduced by $\sim \mathrm{x} 2$ with the use of this toroidal mirror but this was viewed as a reasonable trade off considering the flexibility of the beamline allowing 2 experimental end stations.

Three and half meters downstream of the slits is a four crystal monochromator in the +--+ configuration [9]. This arrangement has the property of directing the monochromatic xrays along the same axis as the incoming white light. The monochromator crystals to be used are 2 identical channel cut crystals mounted such that the rotation axis passes through the surface of diffracting surfaces 2 and 3. In this manner a Bragg angular range of 7-70 degrees is allowed whilst the channel cut crystals themselves remain compact having a total length of $59 \mathrm{~mm}$. For $\mathrm{Ge}(111)$ monochromator crystals the angular range corresponds to an energy

range of $15574-2019 \mathrm{eV}$ which is a reasonable match to the cut off of the toroidal mirror 
$(\sim 50 \%$ reflectivity at $14 \mathrm{KeV})$. The off axis rotation of the crystals allows for them to be rotated out of the way and thus allow white radiation to continue to the KB focusing mirrors. The 4-crystal monochromator is of a design similar to that of Tolentino et al [10] consisting of two rotational stages onto which two channel cut crystals mount. For Bragg angle changes the two stages rotate in opposite directions by means of a tape drive, which is driven by a linear slide. We find the instrument is able to scan in energy and remain on the rocking curve over the available photon energy range of $6-15 \mathrm{KeV}$ without the additional requirement of feedback.

The KB mirror dimensions are defined by the source size, operating wavelength, spot size at the sample and working distance required around the sample. It is useful to standardize on one design for both the horizontal and vertical focusing KB mirrors. The maximum source size is defined by the focus of the toroidal mirror at $250 \times 45 \mu \mathrm{m}$. A design spot size at the sample of $0.5 \mu \mathrm{m}$ would be appropriate for the electromigration work. The operating wavelength should extend to $\sim 14 \mathrm{KeV}$ to ensure an adequate number of Laue reflections. This implies a grazing incidence angle slightly less than the 5.4mrads grazing angle of the toroidal mirror to avoid the rapid flux reduction at high energies due to multiple reflections near the critical angle. The maximum convergence angle of the light onto the sample is limited by the critical angle of reflection of the highest energy required. If a higher convergence angle is used it means that at one end of the mirror light will be traveling almost parallel to the surface and at the other it will be exceeding the critical angle and will not be reflected. For $14 \mathrm{KeV}$ photons the critical energy reflected from a platinum mirror is about $5.4 \mathrm{mrad}$. To avoid this critical angle issue a convergence angle onto the sample of less than $5.4 \mathrm{mrad}$ is required. This restricts the aperture of the KB mirrors. We also arrange for the vertical focusing $\mathrm{KB}$ mirror to be just before the sample. This has the effect of reducing the vertical aperture accepted such that less of the toroidal mirror surface length is used. This will reduce the effect of the brightness reduction due to tangential errors of the toroidal mirror. The four-crystal monochromator also has a vertical angular acceptance limited by the convolution of 3 single crystal-rocking curves. Fig. 3 shows the variation in vertical angular acceptance with change in photon energy - at $10 \mathrm{KeV}$ the angular acceptance is $~ 55$ urads FWHM.

Taking the above factors into consideration we opted for KB mirrors of length $=101.6 \mathrm{~mm}$, a grazing angle $=4.5 \mathrm{mrads}$ and a sample to mirror end clearance distance of $60 \mathrm{~mm}$. Other parameters are listed in Table 1. For the photon energies used here $(>6 \mathrm{KeV})$ where the vertical acceptance is strongly limited by the monochromator (fig.3) we stop the vertical aperture down to $\sim 65 \mu \mathrm{rads}-$ about half the $\mathrm{KB}$ mirror aperture. A collimating mirror before the monochromator will eliminate this vertical aperture restriction by the monochromator and is planned.

The KB mirrors are required to be plane ellipses in order to faithfully image the adjustable slit source to the sub micron focus at the sample. In general only spheres and flats can be manufactured by the optics industry to the sub micro-radian tolerance required. The manufacturing of plane ellipses to sub micro-radian tolerances is beyond the current state of the art. To make plane ellipses to the relevant tolerance we bend flat mirrors in a mechanical mirror bender. The schematic layout of the device used is shown in fig.4. It consists of a flat mirror of dimensions $101.6 \times 12.7 \times 5 \mathrm{~mm}$ thick with invar blocks attached to the mirror ends with epoxy [11]. It is important to attach the blocks to the ends of the mirror rather than the mirror base as experience indicates [12] that the later sets up longitudinal strain within the mirror resulting in significant mirror surface deformation. The blocks are bolted to weak 
springs that are attached to slide ways that are driven by pico-motors. Moving the slide ways apart results in the leaf springs adopting a ' $S$ ' shape which provide the required bending moment for the mirror ends. The mirror is constrained from translating to the side by means of a thin strip of metal labeled 'tie bar' in fig. 4. One of the merits of applying couples through weak springs is that the extension of the spring can be large, so the couples can be applied with high accuracy using relatively coarse actuators. In addition, the change in couple caused by thermal expansion mismatch of the mirror to the base is virtually eliminated. Expressing the moment in terms of the applied couples, the simple beam bending equation can be rewritten as,

$$
E I_{0} \frac{d^{2} y}{d x^{2}}=\frac{\mathrm{C}_{1}+\mathrm{C}_{2}}{2}-\frac{\mathrm{C}_{1}-\mathrm{C}_{2}}{\mathrm{~L}} x
$$

where $\mathrm{E}$ is the modulus of elasticity, $\mathrm{I}$ is the moment of inertia of the beam, $\mathrm{C}_{1}$ and $\mathrm{C}_{2}$ are the applied couples, $\mathrm{y}$ the vertical displacement, $\mathrm{x}$ the distance along the beam from the middle and $\mathrm{L}$ the beam length. The sum of the couples is responsible for a fixed curvature term. The difference in the couples is responsible for a term that varies linearly with displacement along the mirror. Having asymmetric couples acting on a plane mirror produces a mirror shape that is a good cubic approximation to the required elliptical mirror shape. To achieve a better fit to the required ellipse the moment of inertia of the beam is varied as a function of $x$. The moment of inertia is given by $\mathrm{I}=\mathrm{bh}^{3} / 12$ where $\mathrm{b}$ is the beam width and $\mathrm{h}$ the thickness. The mirror is fabricated with its width varying along its length. This allows the flat mirror to be bent to the exact plane ellipse required. Details of this approach and the fundamental beam bending calculations can be found in [13].

The mirror shaping required for these mirrors is most severe for the last $\mathrm{KB}$ mirror that is required to provide the maximum demagnification. The width varies from $7.87 \mathrm{~mm}$ at the upstream end, $12.7 \mathrm{~mm}$ in the middle and $9.17 \mathrm{~mm}$ at the downstream end. During the assembly the mirrors are de-twisted when approximately unbent and flat using a Zygo Mark 4 interferometer and then shaped on a long trace profiler [14]. Fig.5 shows a typical trace of the figure error recorded with slope errors of $0.6 \mu \mathrm{rad}$ over the middle $80 \%$ of the mirror. Spot sizes are typically $\sim 0.9 \mu \mathrm{m}$ as shown in fig.6. For the demagnifications used, the vertical mirror is performing about $\mathrm{x} 3$ worse than designed. The horizontal mirror is performing at its designed spot size.

The local geometry around the sample is shown schematically in fig.7. The sample is inclined at approximately 45 degrees to the white x-ray beam. The Laue diffraction pattern is collected by the x-ray CCD camera located $\sim 30 \mathrm{~mm}$ above the sample. The sample can be heated up to $\sim 400^{\circ} \mathrm{C}$ by a heater attached to the back face of the semiconductor chip. The whole sample assembly is mounted on a piezo stage that has a range of $\pm 50 \mu \mathrm{m}$ in the plane of the sample. This in turn is mounted off the XYZ motorized mount of the goniometer head. This has a range of $\pm 10 \mathrm{~mm}$ and allows for coarse adjustment.

The electromigration samples investigated were pure Al two level test structures 10 to $100 \mu \mathrm{m}$ long, $0.7 \mu \mathrm{m}$ wide and $0.75 \mu \mathrm{m}$ thick. Shunt layers of Ti cover the top and bottom of the lines. The lines are buried under $0.7 \mu \mathrm{m}$ of silicon dioxide for insulation. The test lines are connected to aluminum pads by tungsten vias of thickness $2250 \AA$. To determine the location of the sample we carry out an x-ray fluorescence map by rastering the sample in front of the focused white beam and detecting the $\mathrm{Ti} \mathrm{K} \alpha$ fluorescence $\mathrm{x}$-ray with a solid-state detector. Having located the sample interconnect line a Laue pattern can be recorded with a $\sim 10 \mathrm{sec}$ 
exposure. Fig.8a shows a typical Laue pattern and is dominated by the bright spots of the silicon substrate. However as indicated by the arrows, weaker spots are also apparent in fig.8a. These are from the single crystal aluminum grain of the interconnect. The silicon spots can be digitally subtracted to yield the aluminum grain Laue pattern shown in fig. $8 \mathrm{~b}$.

Distance and CCD center channel calibration was determined by moving the CCD camera radially from the sample and recording the silicon Laue patterns at various distances from the sample. Lines drawn through the succession of the same Laue spots intersect at the sample point and this defines the sample position relative to the CCD x-ray detection surface. Using the silicon Laue pattern as a reference allows for detailed calibration of the roll, tilt and yaw of the CCD x-ray detection surface. Custom code using an algorithm similar to the one described by Chung et al [15] is able to automatically index the Laue pattern using the $\mathrm{CCD} /$ sample geometry and the known crystal structure of aluminum. The code achieves this by determining the inter-ray angles for the Laue spots and comparing these to the various calculated values of indexed Laue spots until a match is found. When an indexed match is found, many other spots are naturally indexed (for aluminum about 9-15 spots). Remaining un-indexed spots belong to an adjacent grain as often several grains are illuminated which results in overlapping Laue patterns. The code is capable of dealing with this and has indexed up to $10+$ overlapping Laue patterns.

Automatic indexing of the Laue patterns allows the instrument to determine the orientation of the grains along the length of an interconnect line to within 0.01 degree. This mapping feature is important since electromigration experiments are often performed on single lines where prior knowledge of individual line structures is particularly relevant. For instance, in-situ observations of void formation on passivated lines using high voltage scanning electron microscopy reveal that voids nucleate at grain junctions [3]. It is particularly important to have a priori knowledge of the grain structure in a line in order to relate void formation to particular grain boundaries and misorentations between grains.

As an example of the detailed mapping capability we show in fig.9 the mapping of a $4 \mu \mathrm{m}$ long aluminum grain. The (111) reflection spot is shown in detail in the sequence in the top part of the figure as the micro beam is scanned down one side of the wire in $0.5 \mu \mathrm{m}$ steps. The diffraction spot clearly moves in position and can be interpreted as a sequence of 3 subgrains with slight misorientation. The angular misorientation between them is less than 0.5 degree. Not only is the instrument able to provide the overall grain orientation, it is able to look in detail within grains to show grain sub-structure.

The indexing code is also capable to accurately determine the position of the Laue spot to $1 / 10^{\text {th }}$ of a pixel (pixel size $=87.9 \mu \mathrm{m}$ ). The slight displacement of the spot from the calculated position is able to yield the deviatoric (distortional) part of the strain tensor $[15,16,17]$. This represents the distortion of the basic shape of the unit cell. Strain sensitivities of $2 \times 10^{-4}$ are typical. In order to get the total strain tensor, the determination of the energy of one Laue spot is necessary in order to get the dilatational component of the tensor (for an isotropic compound, this component is directly related to the hydrostatic stress - the volume change of the unit cell). This is done in the following way.

From previous white beam measurements we have determined the angular position or the Bragg angle of the Laue reflections from their pixel coordinates on the CCD. From the indexed Laue pattern, the approximate energies of the reflections are calculated with the hypothesis of unit cell volume conservation. To measure the $\mathrm{d}$ spacing we concentrate our attention on a single Laue spot say (242) and its relevant pixel region on the detector. We now 
insert the 4-crystal monochromator into the path of the white beam and scan energy over the appropriate energy range. In the monochromatic beam experiment the incident spot size is enlarged to about $\sim 10 \times 5 \mu \mathrm{m}$, a procedure that we have found advantageous since it increases the total flux on the sample and allows for parallel recording of the diffraction spots of the sub grains. Exposure times under these conditions amount to a few seconds. From the white beam Laue pattern we know the exact CCD pixel position where the Laue reflection for a particular sub grain is located. An example of an energy scan for the (242) reflection of a $4 \mu \mathrm{m}$ long aluminum grain at $225^{\circ} \mathrm{C}$ is shown in Fig.10. The peaks correspond to different regions (sub grains) within the long grain. Two significant features are noteworthy; (1) the energy range over which different regions of the same grain reflect is $\sim 60 \mathrm{eV}$ and (2) with some variation each fitted peak is about $10 \mathrm{eV}$ wide whereas for perfect aluminum the width should be about $3 \mathrm{eV}$ allowing for the resolution of this instrument. From the variation in the energy and pixel position we can obtain the varying d spacing of the (242) planes along the grain. Furthermore from its variation with location in the grain we can obtain the change in strain or the local strain gradients within a grain. The relative change in strain along the grain length is shown in fig.10(b). From the width of the peaks we can also conclude that each sub-grain is substantially strained. Gradients of strain as large as $3.5 \times 10^{-4} / \mu \mathrm{m}$ in single grains are evident from fig.10b).

Our initial in-situ electromigration experiments were carried out on a passivated aluminum line $100 \mu \mathrm{m}$ in length. The applied current density was $7.6 \times 10^{5} \mathrm{~A} / \mathrm{cm}^{2}$ at a temperature of $200{ }^{\circ} \mathrm{C}$ in order to speed up the phenomenon of electromigration so that experimental observation times are reasonable. Orientation measurements at the ends of the line segment show two grains that extend through the width and depth of the line (termed a bamboo-like configuration). The total hydrostatic stress at the wire ends is shown in fig. 11. We observe a slight hydrostatic stress gradient along the wire length of $0.8 \mathrm{MPa} / \mu \mathrm{m}$ consistent with material removal from the cathode end and deposited at the anode end. We also observe large local stress gradients within the grains of up to $80 \mathrm{MPa} / \mu \mathrm{m}$. These local stress gradients play an important role in the electromigration phenomena, but, to date have been ignored because of the lack of experimental strain data at the micron level.

\section{Conclusion}

In summary, we have developed a new X-ray micro diffraction technique capable of resolving sub-micron variations of texture and strain with an accuracy of about 0.01 degree in orientation and about $2.10^{-4}$ in strain respectively. For aluminum this corresponds to a stress sensitivity of $20 \mathrm{MPa}$. We have successfully applied the technique to in-situ electromigration experiments in sub-micron sized aluminum interconnect test structures which will provide new insights on the key physical parameters during electromigration. Our initial results show large local stress gradients within grains. The observed mosaic structure of the grains indicate that interconnect lines have experienced plastic deformation and creep during processing. We believe that detail on this scale in buried passivated structures has not been reported before.

Further development of the X-ray micro beam technique will provide key information on the behavior of materials at micron and sub micron level. This can provide us with a new and unique approach, which will broadly enhance our understanding of the complex and intricate links between the macroscopic and the microscopic properties of thin and bulk polycrystalline materials. 


\section{Acknowledgements}

The Advanced Light Source is supported by the Director, Office of Science, Office of Basic Energy Sciences, Materials Sciences Division, of the U.S. Department of Energy under Contract No. DE-AC03-76SF00098 at Lawrence Berkeley National Laboratory. We thank Intel for the partial funding of the end station.

\section{References}

1. I. A. Blech and C. Herring, Appl. Phys. Lett. 29 (1976) 131.

2. I. A. Blech and K. L. Tai, Appl. Phys. Lett., 30, (1977) 387.

3. T.Marieb, P.Flinn, J.C.Bravman, D.Gardner and M. Madden, J. Appl. Physics, 78 (1995) 1026.

4. P.C.Wang, G.S.Cargill, I.C.Noyan, E.G.Liniger, C.K.Hu and K.Y.Lee, MRS Proceedings 473 (1997) 273.

5. A.Bruerke, H.Wendrock, T.Kitter, S.Menzel, K.Wetzig and A.V.Glasow. Mat. Res. Soc. Symp. Proc, 563 (1999) 109.

6. D.Hartman, P.A.Heimann, A.A.MacDowell, K.D.Franck, A.P.Grieshop, S.C.Irick and H.A.Padmore. SPIE vol 3447 (1998) 40.

7. P.Kirkpatrick and A.V.Baez, J.Opt.Soc.Am., 38 (1948) 766.

8. SMART 6000 CCD system, Bruker AXS, Madison, WI 5373, USA

9. J.H.Beaumont and M.Hart, J.Phys.E, 7 (1974) 823.

10. H.Tolentino and A.R.D.Rodrigues, Rev.Sci.Instrum. 63 (1992) 946.

11. Hysol EA 9303.3NA epoxy, Dexter Aerospace Materials Div. Pittsburg, CA 94585, USA

12. H. A. Padmore, M. R. Howells, S. Irick, T. Renner, R. Sandler and Y-M Koo, Proc. SPIE, 2856 (1996) 145.

13. M.R.Howells and D.Lunt, Opt. Eng. 32 (1993) 1981.

14. S. Irick, Nucl. Inst. Meth., A347 (1994) 226.

15. J.S.Chung and G.E.Ice, Mat. Res. Soc. Symp. Proc, 524 (1999) 49.

16. C. Noyan and J. B. Cohen, Residual Stress: Measurement by Diffraction and Interpretation (Springer, New York, 1987), p. 33

17. J.-S. Chung and G. E. Ice, J. Appl. Physics, 86 (1999) 5249. 


\section{Figures Captions}

Fig.1 Schematic layout of beamline 7.3.3 at the ALS.

Fig.2. 3D model of the micro-diffraction end station

Fig.3. Vertical acceptance of a 4 crystal Ge(111) monochromator versus photon energy

Fig.4. Schematic layout of the KB mirror bending mechanism

Fig.5 Long trace profile for one of the KB mirrors. The RMS figure error is $0.6 \mu$ rad over the mid $80 \%$ of the mirror

Fig.6. Typical focus spot sizes recorded by differentiating a knife edge test. For the vertical case the source to mirror distance $=3537 \mathrm{~mm}$, mirror to focus $=120 \mathrm{~mm}$, demagnification $=$ 28 , source size $=10 \mu \mathrm{m}$. For the horizontal case the numbers are $3387 \mathrm{~mm}, 270 \mathrm{~mm}, 12.5$ and $10 \mu \mathrm{m}$

Fig.7. Schematic layout around the sample and CCD region. The sample is scanned in its own plane on an XY piezo stage as indicated by the arrows.

Fig. 8. (a). Raw data of the Laue pattern recorded from a single grain aluminum interconnect with a silicon substrate. The pattern is dominated by the silicon pattern, but the weak spots (indicated by arrows) is the Laue pattern from the aluminum grain. (b). The silicon Laue pattern has been digitally subtracted leaving the aluminum Laue pattern that has been indexed.

Fig. 9. Sub-grain structure of a single grain in a $10 \mu \mathrm{m}$ line. The pixel position of the (111) Laue spot as a function of position along the grain is shown in the upper sequence.

Fig. 10 (a) Monochromatic beam scan of (242) reflection showing the broad energy range $(60 \mathrm{eV})$ of this Laue spot. The various sub-grain reflections are shown resolved in this figure. The strain of each sub-grain is indicated beside each of the interpolated peaks. The width of each peak is about $10 \mathrm{eV}$ compared to that for a perfect $\mathrm{Al}$ crystal about $3 \mathrm{eV}$. (b) The strain as a function of position calculated from the energies and pixel positions of the peaks in Fig. 11a. Local variations of the strain gradient exist in a single grain.

Fig. 11. Initial electromigration experiment. Two grains at each end of the $100 \mu \mathrm{m}$ line were monitored throughout the experiment (A,B,C,D). After about 10 hours the hydrostatic stress versus position along the line was measured and is shown in the 2 plots. Significant local variation of the stress is apparent. There are indication of a slight stress gradient from one end of the line to the other as indicated by the absolute magnitude of the stress values at each end. 
Table

\begin{tabular}{|l|l|l|l|l|l|}
\hline & Demag. & $\begin{array}{l}\text { Source size } \\
\text { for } 0.5 \mu \mathrm{m} \\
\text { focus }\end{array}$ & $\begin{array}{l}\text { Mirror - } \\
\text { sample } \\
\text { distance }\end{array}$ & $\begin{array}{l}\text { Maximum } \\
\text { Acceptance } \\
\text { angle }\end{array}$ & $\begin{array}{l}\text { Maximum } \\
\text { Convergence } \\
\text { onto sample }\end{array}$ \\
\hline Vertical focus KB & 28.3 & $14 \mu \mathrm{m}$ & $120 \mathrm{~mm}$ & $131 \mu \mathrm{rads}$ & $3.7 \mathrm{mrads}$ \\
\hline Horizontal focus KB & 12.5 & $6 \mu \mathrm{m}$ & $270 \mathrm{~mm}$ & $132 \mu \mathrm{rads}$ & $1.6 \mathrm{mrads}$ \\
\hline
\end{tabular}

Table 1. Parameters used for the KB mirrors. 


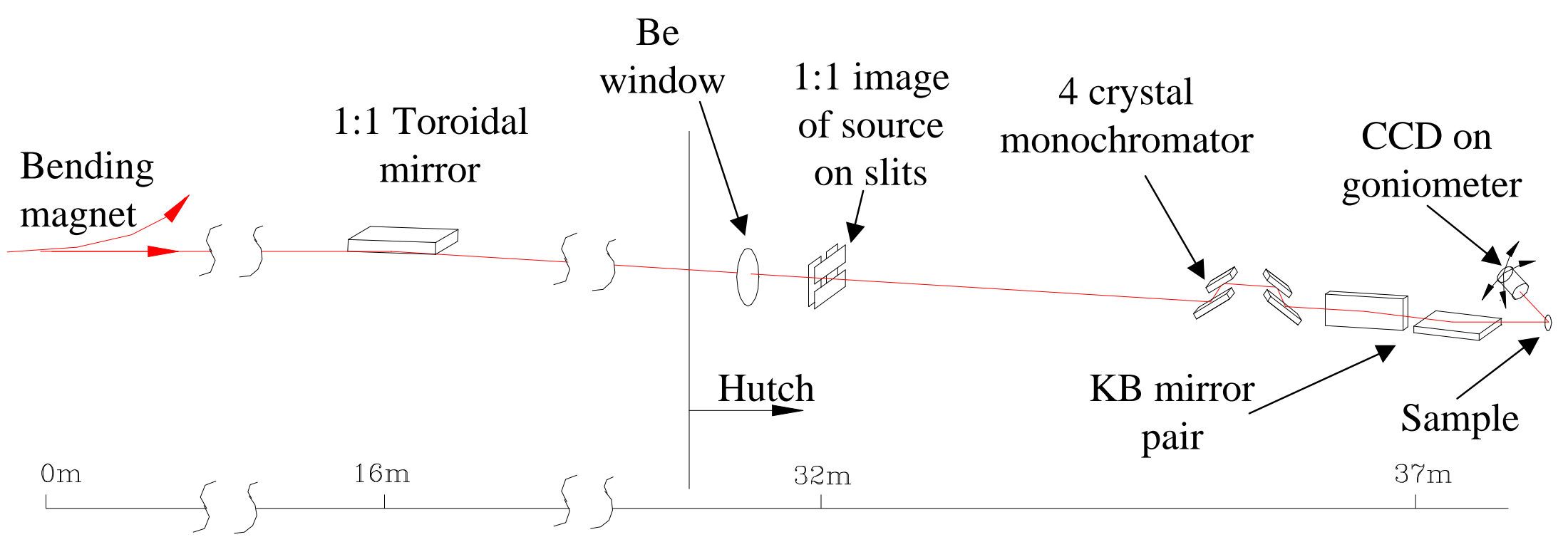

Fig.1 


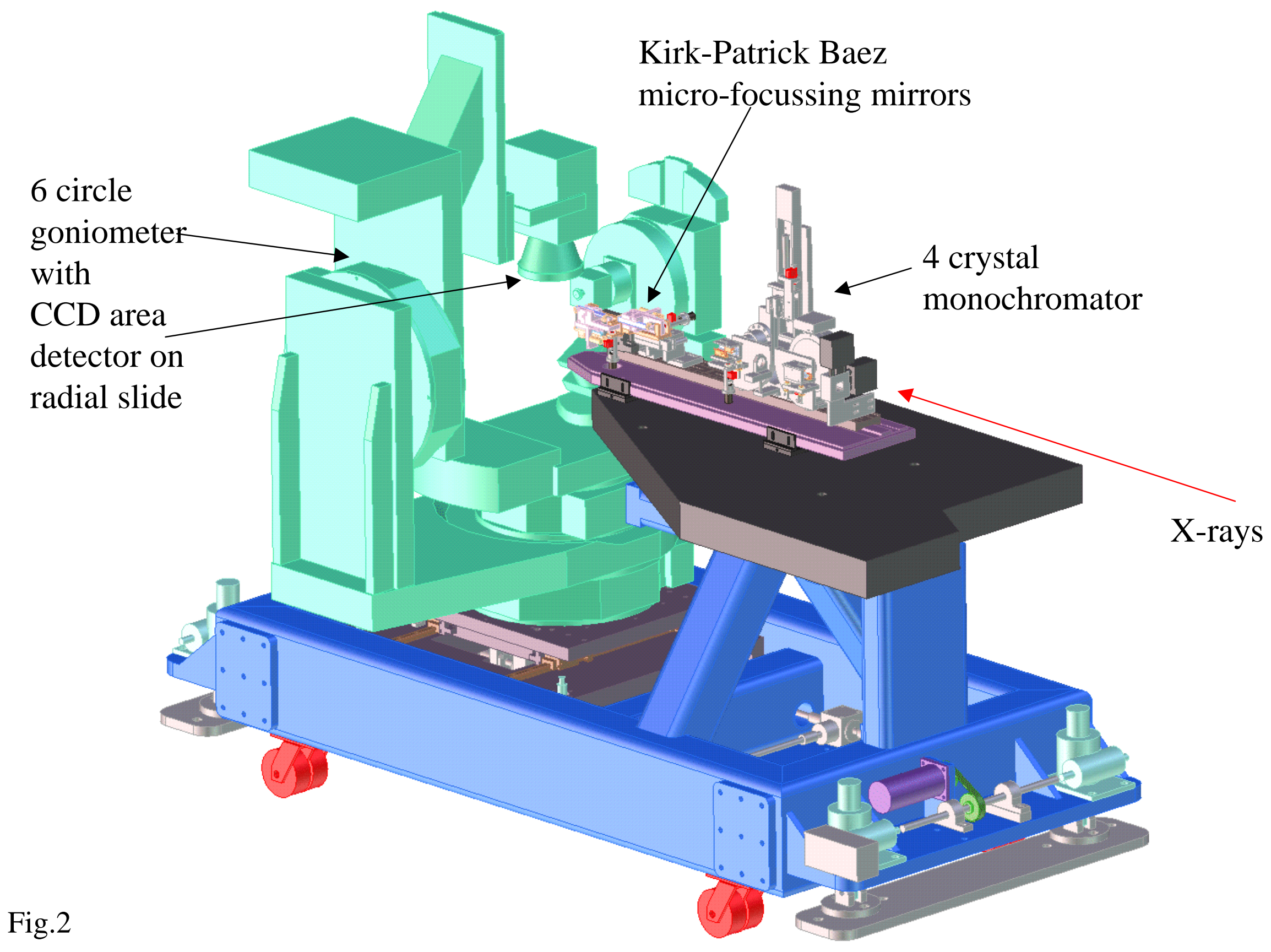




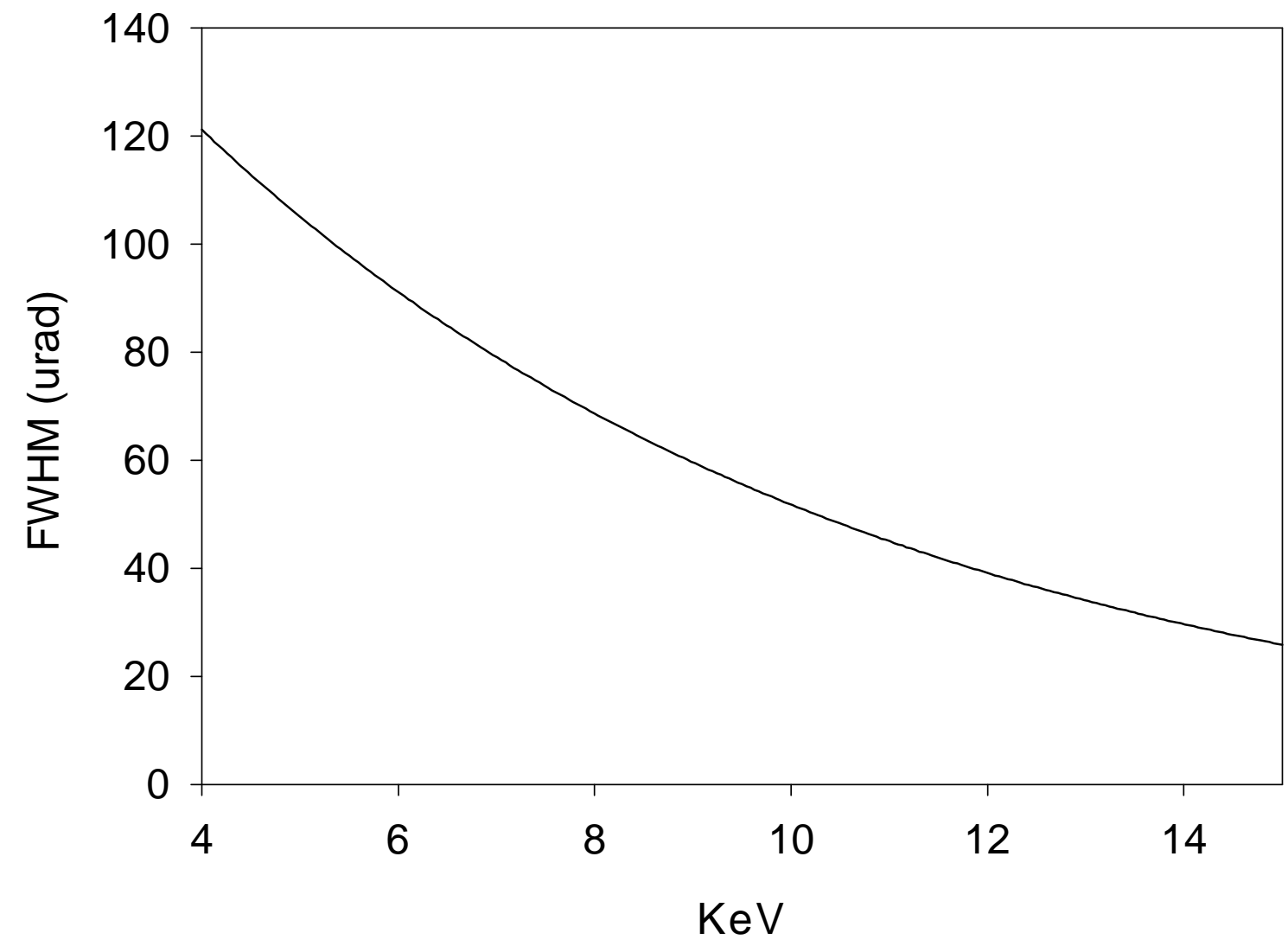

Fig.3 


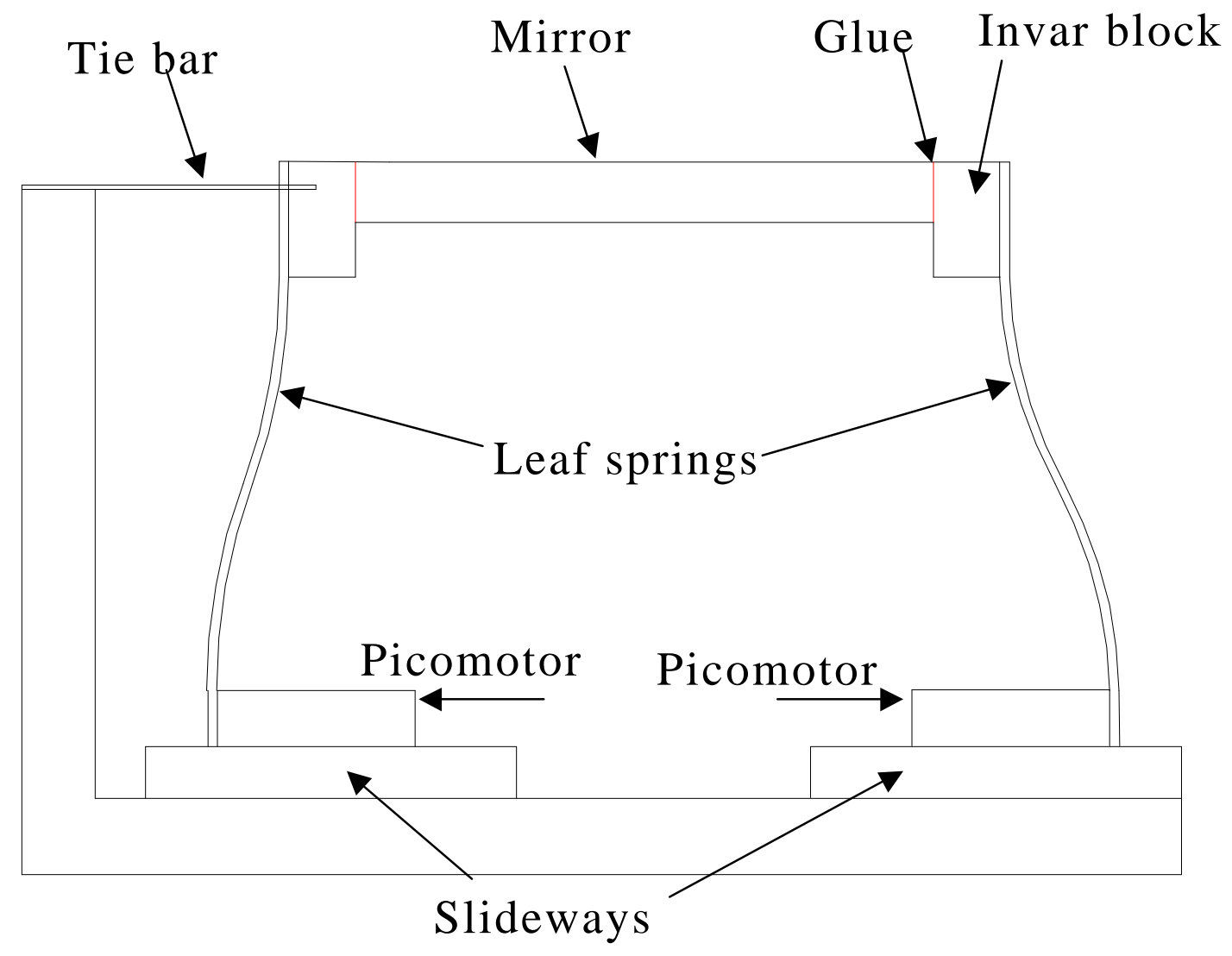

Fig.4 


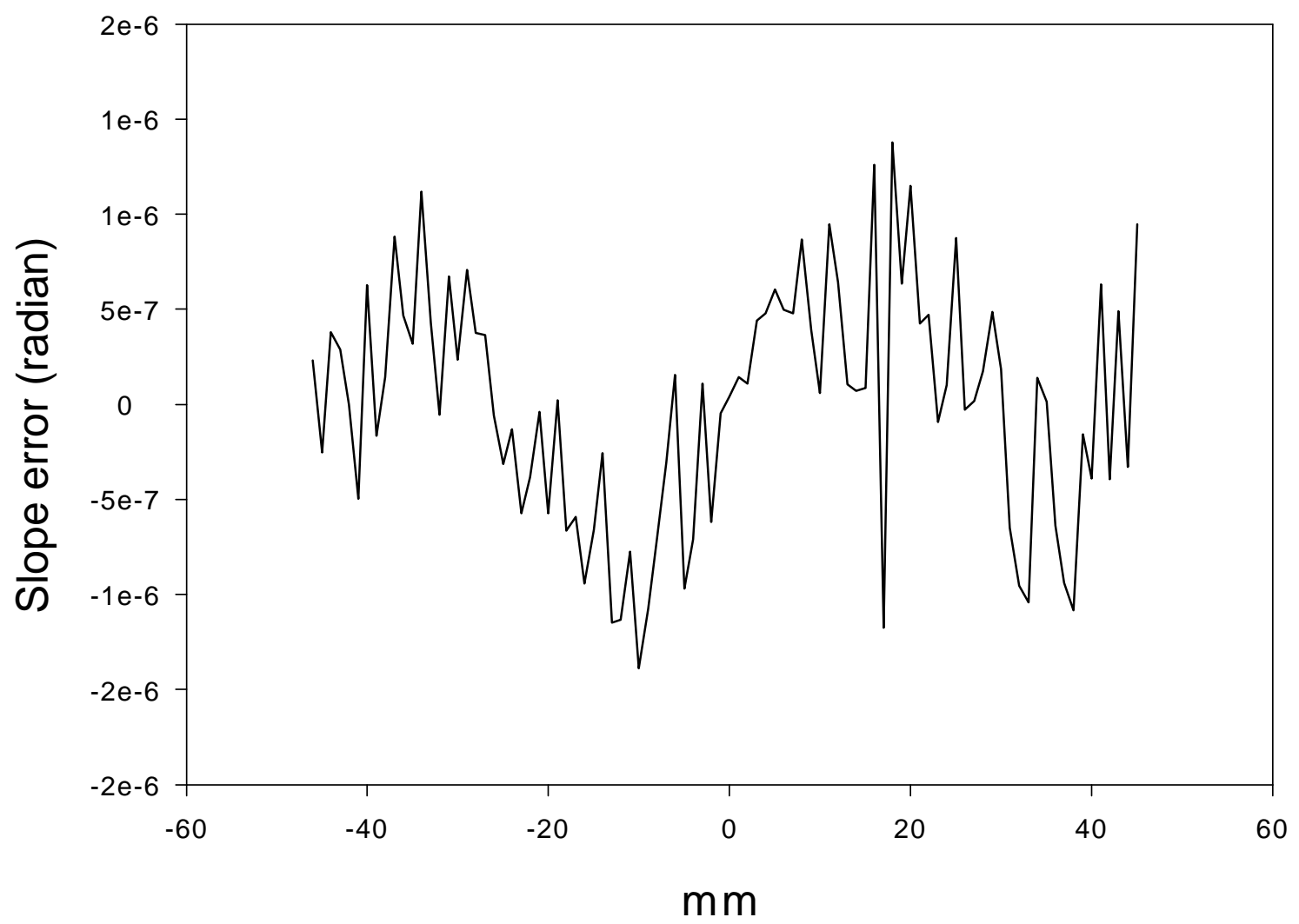

Fig. 5 

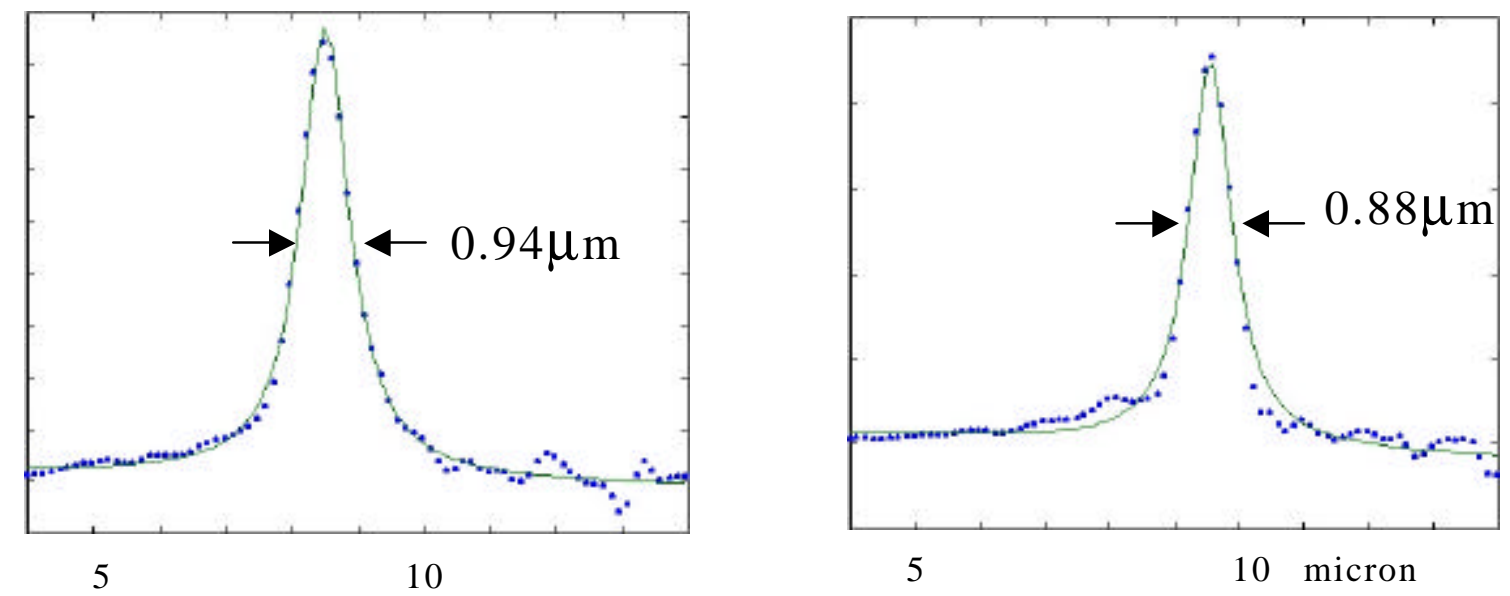

Fig.6 


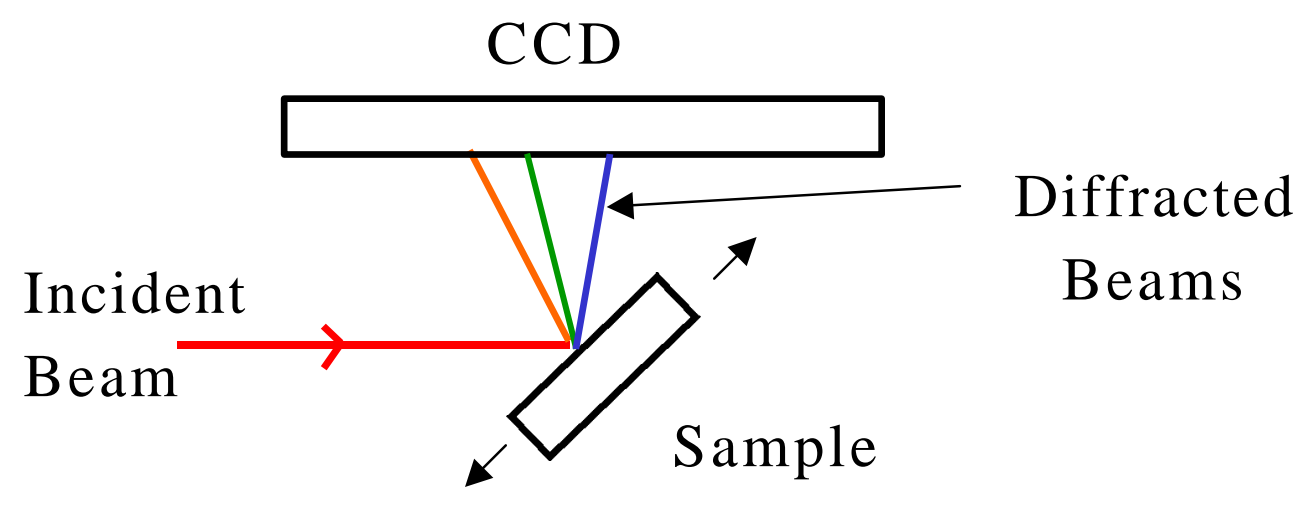

Fig.7. 

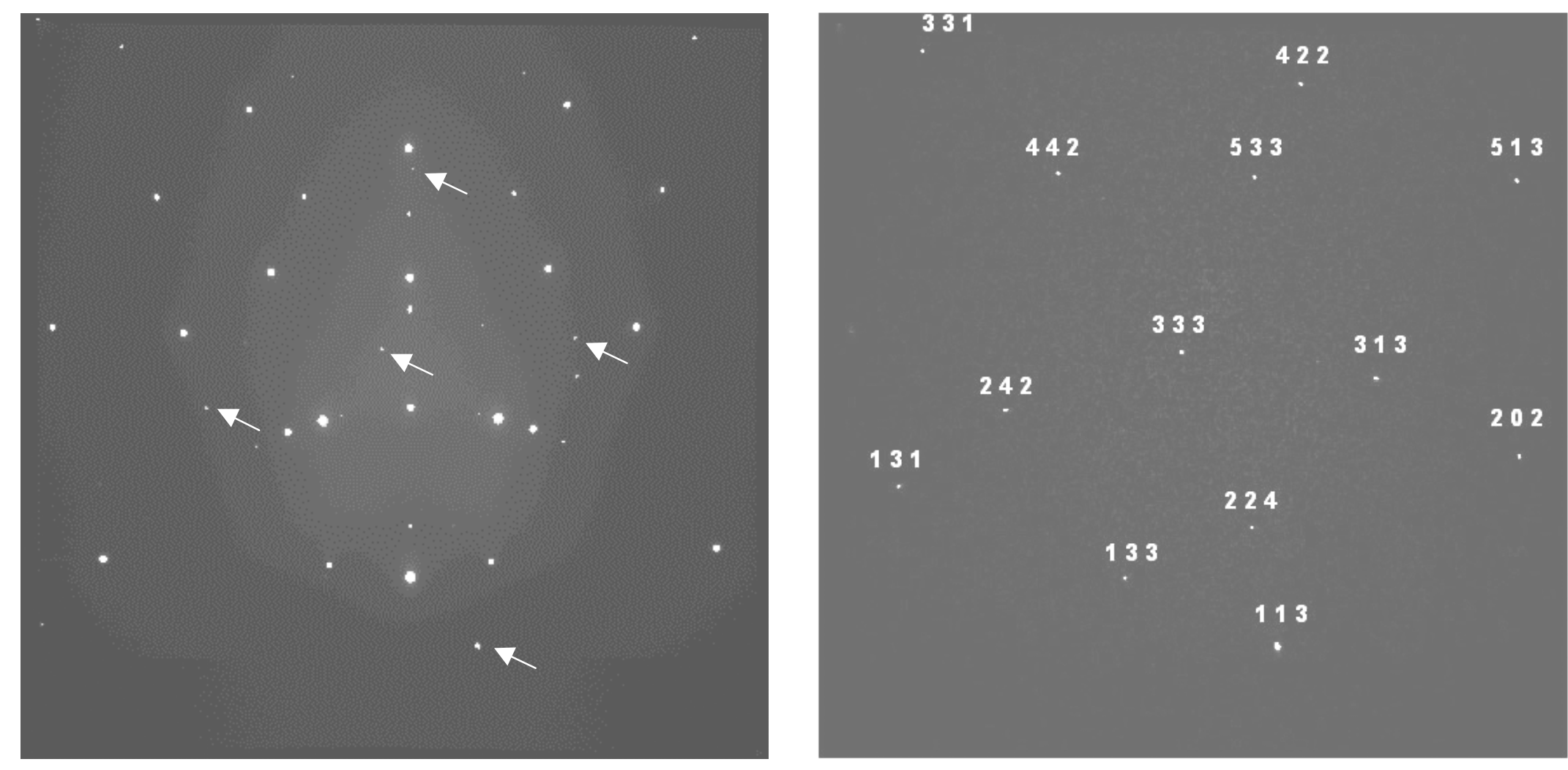

Fig.8. 


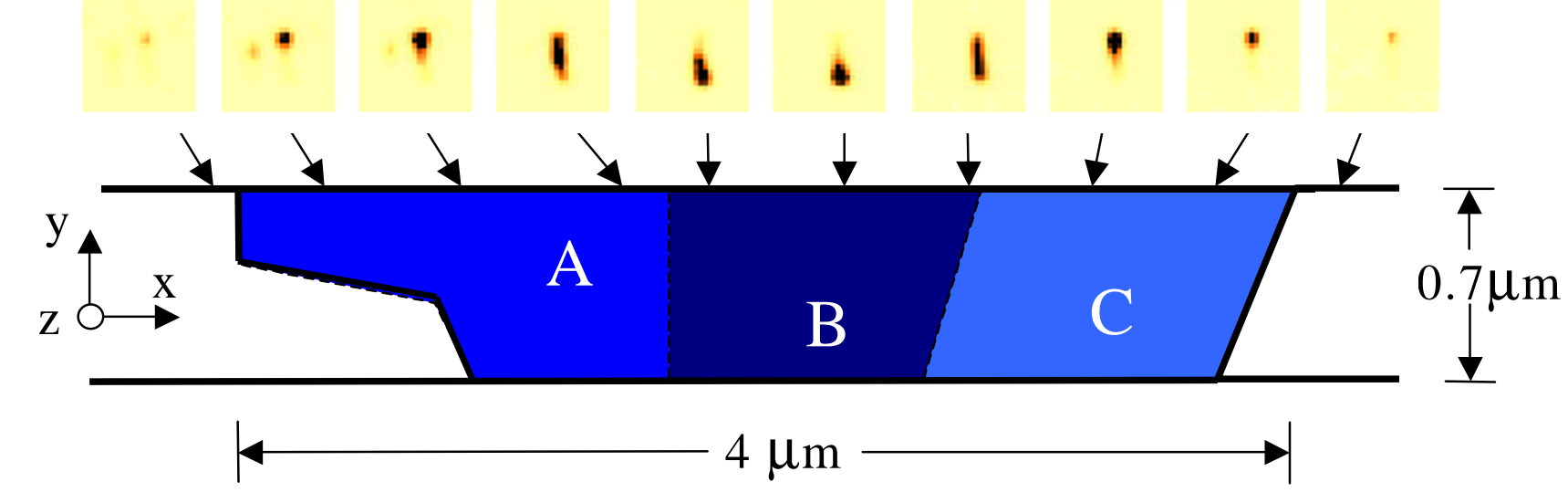

Fig.9 


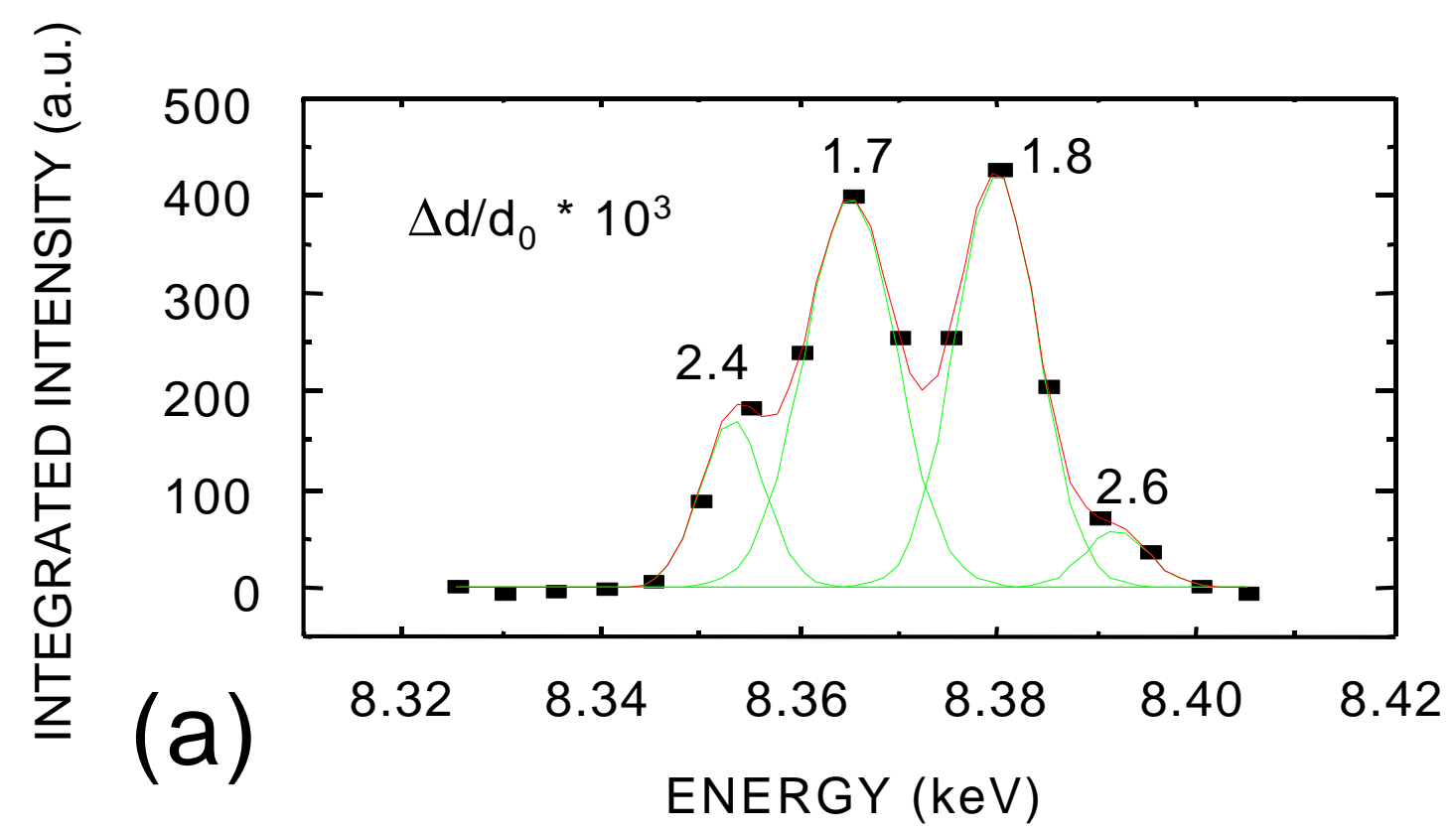

Fig.10

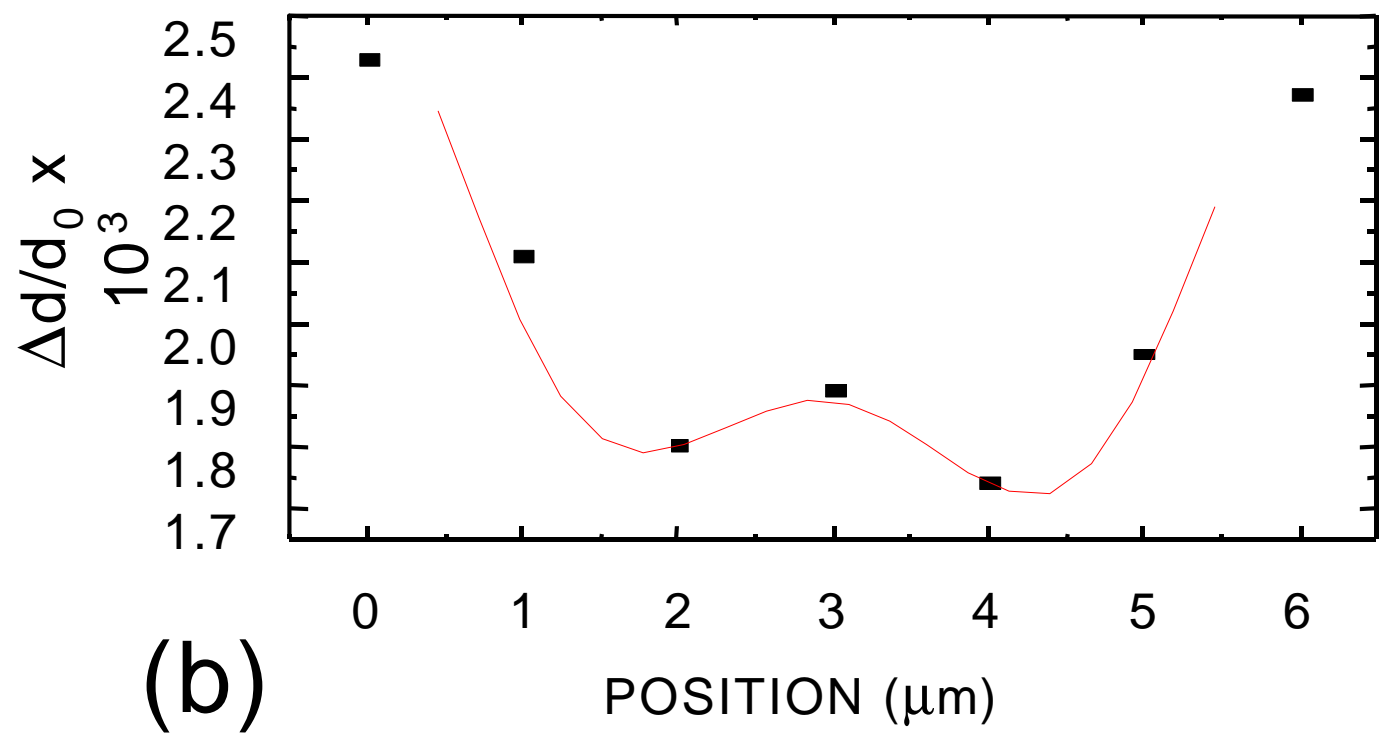



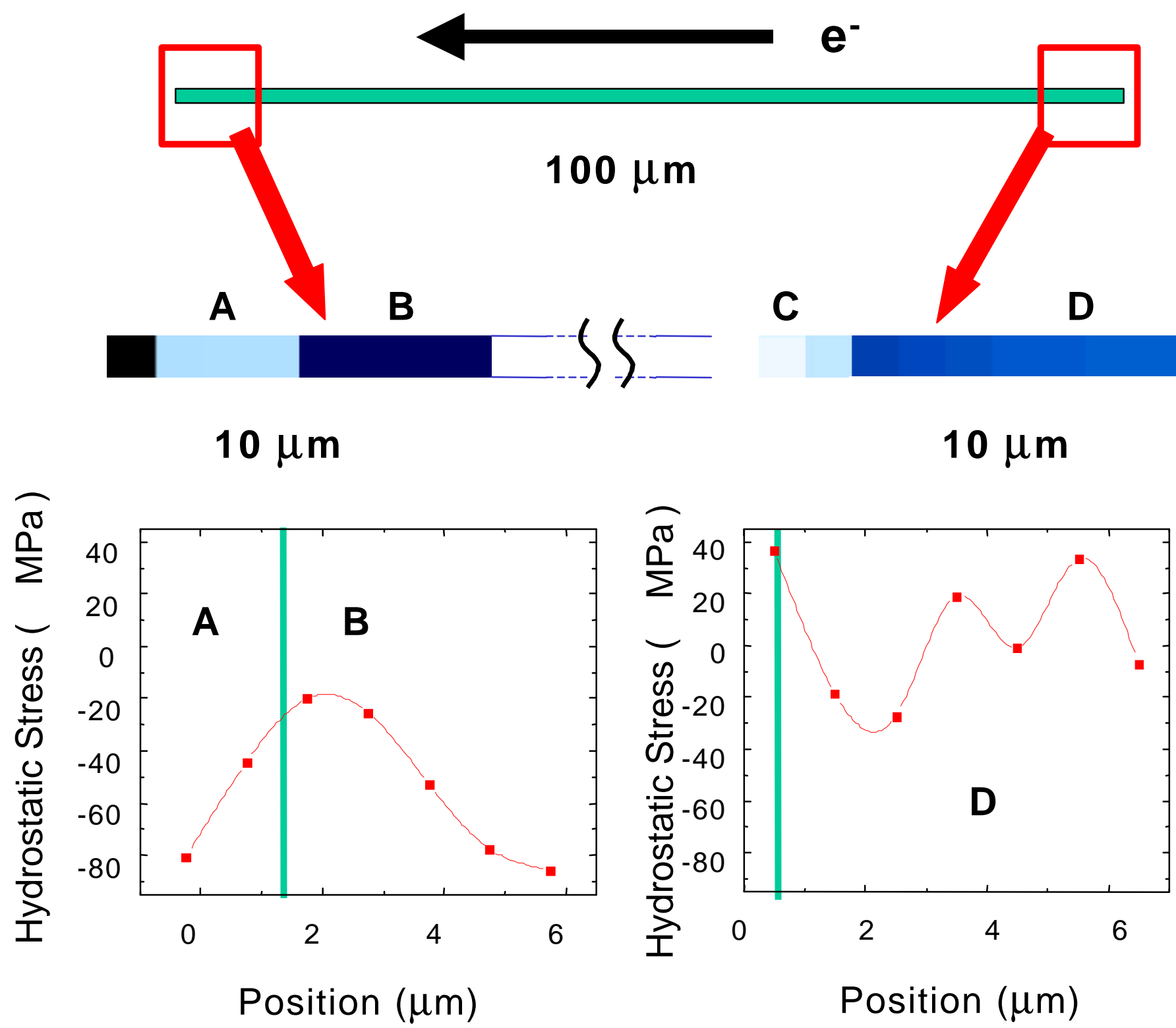

Fig. 11 